\title{
Review
}

\section{Advances in antibiotic therapy for infection after the surgical installation of implants to treat internal fractures}

Yue Sun, Ying Liu, Hongming Zhu

Department of Pharmacy, Sino-singapore Eco-city Hospital of Tianjin Medical University, Tianjin China

\author{
Keywords \\ internal fixation-related infection, osteomyelitis, \\ calcium sulfate, antibiotic therapy

\section{Correspondence} \\ Hongming Zhu, \\ E-mail: zhuhongmingpha@163.com \\ DOI: 10.1515/ii-2017-0133
}

\begin{abstract}
To summarize the advances in antibiotic therapy for infection after the surgical installation of implants to treat internal fractures. Recent studies on antibiotic therapy for infection after the surgical installation of implants to internal fractures were reviewed and analyzed. In general, systematic antibiotics are selected based on the results of bacterial culture. The duration of antibiotic treatment lasts for no more than 4 to 6 weeks. Orally administered and intravenously injected antibiotics have similar efficacies. Orally administered antibiotics exhibit a lower incidence of complications and are less costly than intravenously injected antibiotics. In addition, the efficacy of daptomycin in the treatment of bone infection is problematic. Rifampicin or fluoroquinolone antibiotics should be jointly administered when infection with bacterial biofilms is likely to occur. Calcium sulfate is a typical topically applied antibiotic delivery vehicle that can be completely degraded, with good biocompatibility, bone conduction, and drug release. The rational, systematic, and combined topical application of antibiotics can effectively decrease the recurrence rates of infection after the surgical installation of implants to treat internal fractures and can improve the quality of life of patients.
\end{abstract}

Postoperative infection has become increasingly common with the increasing use of surgery to treat internal fractures. According to the literature, the incidence rate of infection after the surgical installation of implants to treat internal fractures is $0.4 \%$ to $16.1 \%$, with an average of approximately $5 \%{ }^{[1]}$. The incidence of infection after the surgical treatment of closed internal fracture is $0.5 \%$ to $2 \%{ }^{[2]}$, whereas that after open fracture is $1 \%$ to $55 \%{ }^{[3]}$. The more severe the injury, the higher the risk of infection. Infection after the surgical treatment of internal fractures is also one of the most serious challenges that orthopedic surgeons face. The difficulty in controlling infection lies in the complex, difficult treatment and high recurrence rates of infection. Antibiotics have a vital role in the treatment of infection after implantation surgeries. The early and judicious use of antibiotics can significantly decrease the recurrence rate of infection and improve the quality of life of patients. This paper provides a review of the recent advances in antibiotic therapy for post-operative infection after the surgical installation of implants to treat internal fractures.

\section{Classification of infection after the surgical installation of implants to treat internal fractures}

\section{Based on the source of infection ${ }^{[2]}$}

Perioperative period infection: infection caused by wound contamination or surgery;

Adjacent lesions: infection caused by penetrating trauma, such as in open fractures and adjacent lesions;

Hematogenous transmission: infection caused by distant tissue or organ infection and transmitted through blood flow or lymph.

Based on the time of occurrence of postoperative infection [1-2] 
Early infection: acute phase. Infection occurs 2 to 3 weeks after surgery and is mostly caused by the infection of wounds or surgical incisions. The pathogens are mostly virulent microorganisms, such as Staphylococcus aureus and Gram-negative bacilli.

Delayed infection: subacute phase. Infection occurs 2 to 10 weeks after surgery and is caused by trauma or internal fixation. During the period, the majority of bacteria begin to form a stable biofilm.

Chronic infection: chronic phase. Infection occurs 10 weeks after surgery. The pathogens are mostly hypovirulent microorganisms, such as coagulase-negative staphylococci. Sometimes the infection is transmitted through blood. Infection mainly manifests as chronic osteomyelitis.

\section{Cierny-Mader type of chronic osteomyelitis (1984) ${ }^{[4]}$}

This type consists of the anatomical and host types.

Based on the extent of infection, the anatomical type can be divided into four sub-types: intramedullary, superficial, circumscribed, and dispersive.

Based on the host's immune status, the host type can be divided into three sub-types: type A for healthy patients who exhibit no other infection except for the osteomyelitis lesion itself; type B for patients with jointly influential wound healing disease, including local lesions and systemic disease; and type $\mathrm{C}$ for patients with immunodeficiency or similar health problems.

\section{Advances in systemic antibiotic therapy}

\section{Time and method of therapy}

Clinical guidelines for the traditional treatment method recommend 6 weeks of antibiotics administered via intravenous injection when infection is combined with osteomyelitis ${ }^{[5]}$. Recent studies, however, have shown that as long as the pathogen is sensitive to antibiotics, the efficacy of intravenous injection is similar to that of oral antibiotics, and that oral antibiotics have fewer side effects and are less costly than intravenously injected antibiotics [5-6]. In addition, no sufficient evidence exists that administering antibiotic therapy for more than 4 to 6 weeks benefits the patient. Recently, the results of a multicenter, prospective, and randomized controlled study has also shown that 6 weeks of antibiotic therapy is sufficient for osteomyelitis caused by diabetes and that the use of antibiotics should not be prolonged ${ }^{[7]}$.

\section{Selection of systematic antibiotics}

The penetration and coverage of antibiotics in bone tissue are key factors in the effective treatment of bone infections ${ }^{[8]}$ and are important reference factors for the selection of antibiotics. In addition, the selection of antibiotics for sensitive pathogens is also important. $S$. aureus and $\beta$-hemolytic streptococcus dominate the acute phase of infection ${ }^{[9]}$. The use of $\beta$-lactam antibiotics alone or combined with aminoglycoside antibiotics will achieve satisfactory results in most patients ${ }^{[10]}$. When MRSA infection is suspected, glycopeptide antibiotics or daptomycin should be preferentially administered and can also be used together to improve the efficacy of rifampicin. One important feature of the sub-acute and chronic phases of infection after internal fixation surgeries is the formation of bacterial biofilms. The pathogenesis of bacterial biofilms has two main aspects: (1) Antagonize immunity, thus causing chronic infection. (2) The special structure of bacterial biofilms requires a high minimum concentration of antibiotics for treatment. The required minimum concentration for bacterial biofilms can reach 10 to 1,000 times that for planktonic bacteria. Many bacteria and fungi can form biofilms. The most common biofilmforming bacterial species include $S$. aureus, S. epidermidis, type-A streptococci, and Pseudomonas aeruginosa; yeast can also form biofilms. ${ }^{[11]}$. Rifampicin and rifamycin are the two antibiotics that can effectively clear bacterial biofilms formed by Staphylococcus species ${ }^{[12]}$. Fluoroquinolone antibiotics can effectively remove biofilms formed by Gram-negative bacteria ${ }^{[13]}$.

Daptomycin has attracted increased research attention. Many studies have reported its efficacy in the treatment of bone infections. Seaton et al. ${ }^{[14]}$ conducted a multi-center, retrospective study that involved 220 patients. Their results showed that daptomycin effectively treats osteomyelitis and is well tolerated in patients; it may be more effective combined with surgical intervention. Through a casecontrol study, Liang et al. ${ }^{[15]}$ found that the clinical efficacy and tolerability of daptomycin are equivalent to that of vancomycin in the treatment of MRSA-induced bone and 
joint infection. Therefore, the replacement of vancomycin by daptomycin in the treatment of bone infections caused by MRSA is recommended if the patient cannot tolerate vancomycin. A randomized controlled clinical study by Byren et al. ${ }^{[16]}$ found that in the treatment of prosthesis infections caused by $S$. aureus, the efficacy of the continuous use of $6 \mathrm{mg} / \mathrm{kg}$ or $8 \mathrm{mg} / \mathrm{kg}$ daptomycin for 6 weeks is similar to those of traditional antibiotics, such as vancomycin, teicoplanin, and semi-synthetic penicillin, and that using daptomycin is safe. In addition to systemic use, daptomycin can also be loaded on antibiotic delivery vehicles for topical anti-infective treatment. Rosslenbroich et al. ${ }^{[17]}$ combined daptomycin with traditional polymethylmethacrylate (PMMA) bone cement for the topical application of daptomycin after the surgical treatment of fractions and complicated osteomyelitis. The combined methomycin and PMMA retain their antimicrobial properties and the structural characteristics of the PMMA delivery vehicle are not compromised. Therefore, daptomycin could be used in combination with antibiotic delivery vehicles for the topical treatment of bone infections. However, given the limited clinical reports on daptomycin, its clinical efficacy in the treatment of bone infection still requires further in-depth study.

\section{Research on delivery vehicles for topical antibiotics}

Antibiotics are mainly applied in the treatment of chronic osteomyelitis. Topical bone ischemia, sclerosis, and softtissue scarring occur due to chronic osteomyelitis, and thus the blood supply is inadequate at the lesion site. Sometimes, systemic antibiotics do not reach the effective sterilization concentration; this effect is often combined with bone nonunion. Bone defects often occur after surgical debridement to remove sequestra. Therefore, the topical application of the appropriate antibiotic delivery vehicle can repair the defect, close the dead cavity, prevent the formation of cicatrix, and kill or inhibit bacteria.

PMMA bone cement is the most commonly used antibiotic delivery vehicle ${ }^{[18]}$. Given its poor biocompatibility and low release rate, it cannot self-degrade in the body and must be removed via surgery. Therefore, the shortcomings of PMMA necessitate the research and development of a highly efficient and biodegradable antibiotic delivery vehicle. Calcium sulfate can meet the requirements for a biodegrable antibiotic delivery vehicle: good bio-compatibility, complete degradation, and capability for bone conduction and drug release. In addition, calcium sulfate will not produce excessive heat when mixed with solutions, thus effectively preserving antibiotic activity ${ }^{[19]}$. In recent years, many studies have compared the relevant characteristics of calcium sulfate as an antibiotic delivery vehicle with those of PMMA. The results of an in vitro experiment conducted by McConoughey et al. ${ }^{[20]}$ showed that the topical antimicrobial capacity of calcium sulfate is similar to or even better than that of PMMA. In a clinical randomized controlled trial that compared the efficacy of PMMA and calcium sulfate as topical antibiotic delivery vehicles in the treatment of chronic osteomyelitis and infected nonunion, McKee et al. ${ }^{[21]}$ found that the efficacy of calcium sulfate is similar to that of PMMA in the removal of infection. However, because calcium sulfate is biodegradable, the required number of operations for the removal of calcium sulfate is less than that for the removal of PMMA. An animal experiment by Beenken et al. ${ }^{[22]}$ showed that as an antibiotic delivery vehicle, chitosan-coated calcium sulfate improves the release of sustained-release drugs and exhibits satisfactory efficacy in the treatment of osteomyelitis. Calcium sulfate does have shortcomings. Guler et al. ${ }^{[23]}$ pointed out that calcium sulfate is weaker than PMMA. Nevertheless, in recent years, the Traumatic Orthopedics Department of Nanfang Hospital has used calcium sulfate as an antibiotic delivery vehicle for gentamicin or vancomycin and in combination with bone transport for the treatment of chronic osteomyelitis and infected nonunion. The use of calcium sulfate in these cases has been highly effective.

In addition, many studies have reported the efficacy of other types of antibiotic delivery vehicles in the treatment of osteomyelitis. Cui et al. ${ }^{[24]}$ developed a novel injectable borate bioactive glass cement loaded with vancomycin. The results of an in vitro test showed that the cure rate of this delivery vehicle in osteomyelitis is $87 \%$. Similarly, an in vitro test by Ding et al. ${ }^{[25]}$ proved that boric acid cement loaded with vancomycin and calcium sulfate loaded with vancomycin are superior to simple intravenous injection 
in the treatment of MRSA-induced osteomyelitis, and that borate bone cement has good biocompatibility and capability to promote bone regeneration. Animal experiments and clinical trials by Bhattacharya et al. ${ }^{[26]}$ showed that the use of porous hydroxyapatite stent loaded with ceftriaxone and sulbactam sodium can effectively treat osteomyelitis, reduce the recurrence rate of infection, and promote bone regeneration. Animal experiments by Kaya et al. ${ }^{[27]}$ showed that calcium hydroxyapatite loaded with tigecycline is effective in treating osteomyelitis caused by MRSA. Although these studies demonstrated the effectiveness of various antibiotic delivery vehicle systems in the treatment of chronic osteomyelitis, most of the results came from animal experiments. Therefore, the reported results of these clinical trials are limited.

With regard to future trends, Kluin et al. ${ }^{[28]}$ suggested that an ideal antibiotic delivery vehicle for the treatment of osteomyelitis should have good biocompatibility, controlled degradation kinetics, and can load and release any antibiotic under strict conditions (longer times, higher topical concentrations), with preferably level zero release kinetics. Although PMMA is the most commonly used delivery vehicle in the treatment of chronic osteomyelitis, it is not the best delivery vehicle because of its non-degradability and poor release properties. Biodegradable collagen and calcium matrix delivery vehicles can release large amounts of antibiotics within $24 \mathrm{~h}$ after implantation; thus, the prolonged release of antibiotics cannot be guaranteed with these vehicles. In addition, collagen and calcium sulfate can absorb water and promote the formation of hematomas, thus increasing the risk of superinfection. Among the types of polylactic acid and polyglycolic acid (PGLA) delivery vehicles, PLGA exhibits the best release characteristics. All delivery vehicles, however, can produce acidic degradation products that cause bone resorption. Therefore, based on the recommended level zero release kinetic theory and for the prevention of acidic degradation, trimethylene carbonate could replace lactic acid and PLGA.

\section{Other issues related to the treatment of infection after the surgical installation of implants to treat internal fractures}

Although antibiotics are essential for the treatment of infection after the surgical installation of implants to treat internal fractures, the application of systemic and topical antibiotics must be based on the thorough debridement of the infected lesion. Otherwise, antibiotic treatment will not be effective ${ }^{[2,5,10]}$. In addition, the reasonable handling of internal fixation also determines the efficacy of antibiotics in patients; during different infection periods, the disposal strategy of internal fixation is not the same. For acute-phase infection, there is no recommendation based on medical evidence on the retention or removal of the internal fixation. However, most scholars suggest that reservations be made as much as possible in the context of stable internal fixation. The removal of internal fixations is advisable in the following situations: (1) when installed in drug addicts or heavy smokers; (2) open fracture; (3) and intramedullary fixation ${ }^{[29]}$. Therefore, during the acute phase, if the internal fixation of the patient is stable, the anti-infective treatment must be strengthened. In addition, infection indexes must be followed up; if local and systemic infection is effectively controlled, internal fixation can be retained temporarily; otherwise, it should be removed as early as possible. During sub-acute and chronic phases of infection, if the fracture has been healed, internal fixation should be promptly removed. Internal fixation should be retained only when stable, the infection is effectively controlled, and conditions of local soft tissue are good ${ }^{[29]}$.

\section{Summary}

Infection after the surgical installation of implants to treat internal fractures is a complex disease, and multidisciplinary collaboration is beneficial for the treatment of patients ${ }^{[30]}$. Antibiotics are an integral part of the treatment of bone infections. The early and appropriate application of antibiotics can significantly reduce the recurrence rate of infection and improve the quality of life of patients.

\section{Declarations}

\section{Acknowledgements}

No. 


\section{Competing interests}

The authors declare that they have no competing interest.

\section{Authors' contributions}

Y Sun and Y Liu made the literature analysis and wrote, discussed and revised the manuscript of this review. HM Zhu critically analyzed and corrected the manuscript. All authors read and approved the final manuscript.

\section{References}

1 Zimmerli W. Clinical presentation and treatment of orthopaedic implant-associated infection. J Intern Med, 2014, 276(2): 111-119.

2 Trampuz A, Zimmerli W. Diagnosis and treatment of infections associated with fracture-fixation devices. Injury, 2006, 37(2): 59-66.

3 Hogan A, Heppert VG, Suda AJ. Osteomyelitis. Arch Orthop Trauma Surg, 2013, 133(9): 1183-1196.

4 Cierny G, Mader JT, Penninck JJ. A clinical staging system for adult osteomyelitis. Clin Orthop, 2003, 414: 7-24.

5 Spellberg B, Lipsky BA. Systemic antibiotic therapy for chronic osteomyelitis in adults. Clin Infect Dis, 2012, 54(3): 393-407.

6 Conterno LO, Turchi MD. Antibiotics for treating chronic osteomyelitis in adults. Cochrane Database Syst Rev, 2013, 9(19): 1292

7 Tone A, Nguyen S, Devemy F, et al. Six-week versus twelve - week antibiotic therapy for nonsurgically treated diabetic foot osteomyelitis: a multicenter open-label controlled randomized study. Diabetes Care, 2015, 38(2): 302-307.

8 Landersdorfer CB, Bulitta JB, Kinzig M, et al. Penetration of antibacterials into bone pharmacokinetic, pharmacodynamic and bioanalytical considerations. Clin Pharmacok, 2009, 48(2): 89-124.

9 Lew DP, Waldvogel FA. Osteomyelitis. Lancet, 2004, 364(9431): 369-379.

10 Senneville E, Nguyen S. Current pharmacotherapy options for osteomyelitis: convergences, divergences and lessons to be drawn. Exp Opin Pharmacother, 2013, 14(6): 723-734.

11 Rao N, Ziran BH, Lipsky BA. Treating osteomyelitis: antibiotics and surgery. Plast Reconstr Surg, 2011, 127(1): 177-187.

12 Trampuz A, Murphy CK, Rothstein DM, et al. Efficacy of a novel rifamycin derivative, ABI-0043, against Staphylococcus aureus in an experimental model of foreign-body infection. Antimicrob Agents Chemother, 2007, 51(7): 2540-2545.

13 Peel TN, Buising KL, Dowsey MM, et al. Outcome of debridement and retention in prosthetic joint infections by methicillin-resistant staphylococci, with special reference to rifampin and fusidic acid combination therapy. Antimicrob Agents Chemother, 2013, 57(1): 350-355.
14 Seaton RA, Viale P, Gargalianos-Kakolyris P, et al. Daptomycin use in patients with osteomyelitis: a preliminary report from the EU-CORE(SM) database. J Antimicrob Chemother, 2013, 68 (7):1642-1649.

15 Liang SY, Khair HN, McDonald JR, et al. Daptomycin versus vancomycin for osteoarticular infections due to methicillin - resistant Staphylococcus aureus (MRSA): a nested case-control study. Eur J Clin Microbiol Infect Dis, 2014, 33(4): 659-664.

16 Byren I, Rege S, Campanaro E, et al. Randomized controlled trial of the safety and efficacy of Daptomycin versus standard-of - care therapy for management of patients with osteomyelitis associated with prosthetic devices undergoing two-stage revision arthroplasty. Antimicrob Agents Chemother, 2012, 56(11): 5626-5632.

17 Rosslenbroich SB, Raschke MJ, Kreis C, et al. Daptomycin: local application in implant-associated infection and complicated osteomyelitis. Scientific World J, 2012, 2012(6): 128-145.

18 Lalidou F, Kolios G and Drosos GI. Bone infections and bone graft substitutes for local antibiotic therapy. Surg Technol Int, 2014, 124: 353-362.

19 Koh I, Lopez A, Helgason B, et al. The compressive modulus and strength of saturated calcium sulphate dihydrate cements: implications for testing standards. J Mech Behav Biomed Mater, 2014, 2014(34): 187-198.

20 McConoughey SJ, Wiseman J, Stoodley P, et al. Comparing PMMA and calcium sulfate as carriers for the local delivery of antibiotics to infected surgical sites. J Biomed Mater Res B Appl Biomater, 2015, 103(4): 870-877.

21 McKee MD, Li-Bland EA, Wild LM, et al. A prospective, randomized clinical trial comparing an antibiotic-impregnated bioabsorbable bone substitute with standard antibiotic-impregnated cement beads in the treatment of chronic osteomyelitis and infected nonunion. J Orthop Trauma, 2010, 24(8): 483-490.

22 Beenken KE, Smith JK, Skinner RA, et al. Chitosan coating to enhance the therapeutic efficacy of calcium sulfate-based antibiotic therapy in the treatment of chronic osteomyelitis. J Biomater Appl, 2014(4) , 4: 514-523.

23 Guler UO, Derincek A, Hersekli MA, et al. Restoration of pull - out strength of the failed pedicle screw: biomechanical comparison of calcium sulfate vs polymethylmethacrylate augmentation. Acta Orthop Traumatol Turc, 2014, 48(2): 202-206.

24 Cui X, Zhao C, Gu Y, et al. A novel injectable borate bioactive glass cement for local delivery of vancomycin to cure osteomyelitis and regenerate bone. J Mater Sci Mater Med, 2014, 25(3): 733-745.

25 Ding $\mathrm{H}$, Zhao CJ, Cui X, et al. A novel injectable borate bioactive glass cement as an antibiotic delivery vehicle for treating osteomyelitis. Plos One, 2014, 9(4): e85472.

26 Bhattacharya R, Kundu B, Nandi SK, et al. Systematic approach to treat 
chronic osteomyelitis through localized drug delivery system: bench to bed side. Mater Sci Eng C Mater Biol Appl, 2013, 33(7): 3986-3993.

27 Kaya M, Simsek-Kaya G, Gursan N, et al. Local treatment of chronic osteomyelitis with surgical debridement and tigecycline - impregnated calcium hydroxyapatite: an experimental study. Oral Surg Oral Med Oral Pathol Oral Radiol, 2012, 113(3): 340-347.

28 Kluin OS, van der Mei HC, Busscher HJ, et al. Biodegradable vs non-biodegradable antibiotic delivery devices in the treatment of osteomyelitis. Expert Opin Drug Deliv, 2013, 10(3): 341-351.

29 Mouzopoulos G, Kanakaris NK, Kontakis G, et al. Management of bone infections in adults: the surgeon's and microbiologist's perspectives. Injury, 2011, 42(5): 18-23.

30 Bauer S, Bouldouyre MA, Oufella A, et al. Impact of a multidisciplinary staff meeting on the quality of antibiotherapy prescription for bone and joint infections in orthopedic surgery. Med Mal Infect, 2012, 42(12): 603-607. 